\title{
At Last! Measurement of fractional statistics
}

\author{
Direct observation of anyonic braiding statistics at the $\nu=1 / 3$ fractional \\ quantum Hall state
}

Authors: James Nakamura, Shuang Liang, Geoffrey C. Gardner, Michael J. Manfra arXiv:2006.14115

\author{
Recommended with a Commentary by Steven A. Kivelson ${ }^{a}$ and \\ Charles M. Marcus ${ }^{b}$, a) Stanford University, Stanford, CA 94205, \\ b) Niels Bohr Institute, University of Copenhagen, and Microsoft \\ Quantum Lab-Copenhagen;
}

The Laughlin liquid - the phase of matter with the topological order responsible for the fractional quantum Hall effect - is theoretically predicted to have quasiparticle excitations with fractional charge [1] and fractional braiding statistics [2]. While in a sense these remarkable properties follow from the same topological order that has been clearly confirmed by precise measurements of the quantized Hall response, it is important to obtain direct evidence of these properties in their own right. Not surprisingly, this undertaking raises new challenges of experimental technique and theoretical interpretation. Indeed, a variety of studies over the past several years have provided convincing evidence of fractional charge from a variety of experiments including measurements of shot noise, interferometry in quantum dots and antidots, and scanning SET measurements.

Experimental measures of the "braid statistics," phases accumulated when one particle encircles another, or, in the case of nonabelian statistics, when three particles are involved in a sequence of surrounds, have proved more challenging, despite the existence of several specific protocols spelled out in the theoretical literature. Recently, a paper on a mesoscopic electron scattering experiment involving colliding transverse beams of electrons in a patterned high-mobility electron gas, has reported scattering indicating fractional statistics in the $\nu=1 / 3$ state [3]. The results are credible but the method and interpretation are somewhat complicated and indirect. The more readily interpreted quantum dot interferometer approach, in which edges surround quasiparticles localized in the interior of the dot, as considered theoretically [4], has not been reported until now; it is the subject of the recommended paper by Nakamura et al.

All proposed interferometric measurements of the quantum numbers of the quasiparticles are based on the relation (Eq. 1 in Nakamura et al)

$$
\theta=2 \pi\left(\frac{e^{\star}}{h c}\right) B A+N \theta^{\star}
$$

relating the adiabatic phase $\theta$ accrued by a quasi-particle on passing around a closed path that encloses an area $A$ and $N$ quasi-particles. Here $e^{\star}$ is the quasiparticle charge and $\theta^{\star}$ 
the statistical angle, which are expected to be $e / 3$ and $2 \pi / 3$ for a $\nu=1 / 3$ Laughlin state, respectively. Thus, measuring these two contributions to the phase would constitute a direct measure of the fractional charge and statistics.

One might ask "what took so long?" High-mobility materials are not new, gate-defined interferometers have been around, and the theory is by now ancient. One reason is a combination of contradictory requirements for mesoscopic interferometers, which necessarily involve tunneling between edges and must also be small enough that excitations remain coherent during their excursion around the device boundary. However, unlike the effective charge, $e^{*}$, which can be measured in the presence of Coulomb blockade, proposed protocols for extracting braid statistics from transport through interferometers do not apply when charging effects dominate, which in small tunneling devices is usually the case.

Edge-state interference effects fall into two regimes, depending on the importance of charging effects [5]. The Aharonov-Bohm (AB) regime, without charging effects, where both $e^{*}$ and braid statistics can be detected, can be distinguished from the Coulomb dominated (CD) regime by the direction of conductance ridges in the 2D plane of gate voltage (controlling occupancy) and magnetic field, termed "pajama stripes" by the Weizmann group.

Small dots, showing strong interference, tend to be Coulomb dominated. A technical development was needed. The Manfra group, experts in growth as well as quantum Hall measurements, solved it with their hybrid expertise: they grew thin capacitor plates using molecular beam epitaxy above and below the high-mobility electron gas, reducing capacitance to allow AB interference to dominate. This technique was demonstrated last year by the same team [6]. The signature of success is that the pajama stripes run in the right direction!

In examining these stripe patterns, the team noticed a pattern of repeatable, but otherwise accidental, jumps in the data, presumably caused by charge rearrangement (i.e. a change in the number of localized quasiparatiicles) within the device. Usually such gatedependent jumps are an annoyance, but the Manfra team noticed a surprising pattern in the data (see Fig. 1): every time the pajama-stripe pattern suffered an accidental jump, the stripe pattern jumped rather precisely one third of the spacing of the stripes, and the pattern repeated over several stripes. (Here "several" is defined as more than a few, but less than many).

Though the jumps were not predicted or designed, the observation is clear: the shift of the pajama pattern in each jump is not random. As anticipated theoretically, this is a clear signature of the abelian braid statistics of the $1 / 3$ state. The work is a tour de force of materials design, device fabrication, measurement, and most of all carefully looking at one's own data.

With the door to $\mathrm{AB}$ interference now open, we anticipate a more controlled version of the experiment, showing similar features but with the number of enclosed quasiparticles tuned by intent. Of course, one would also like to see other fractions as well, especially ones where $1 / 3$ is not the only fraction appearing in the quantized conductance, the effective quasi-particle charge, and the statistical phase at the same time. Observing some of the expected interference patterns in these states would more clearly allow these separate pieces of physics to be disentangled. (We are curious what this device showed at other fractions.) Comparable devices with in-situ epitaxial capacitors, a key enabling innovation, as well as interior floating gates [8] hold great promise. It is easy to imagine a path from here to 
(a)

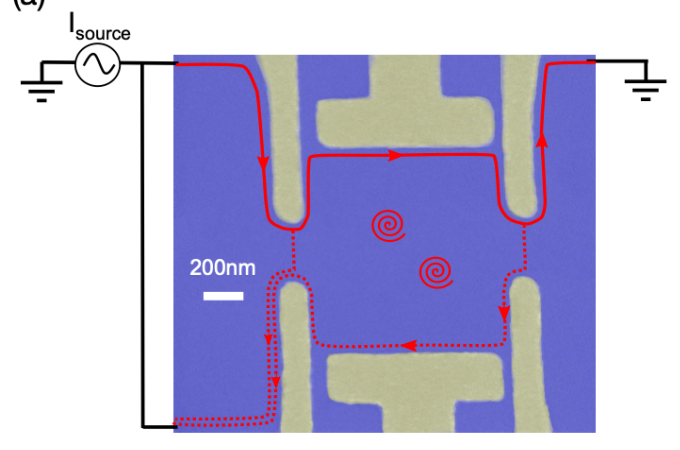

(b)

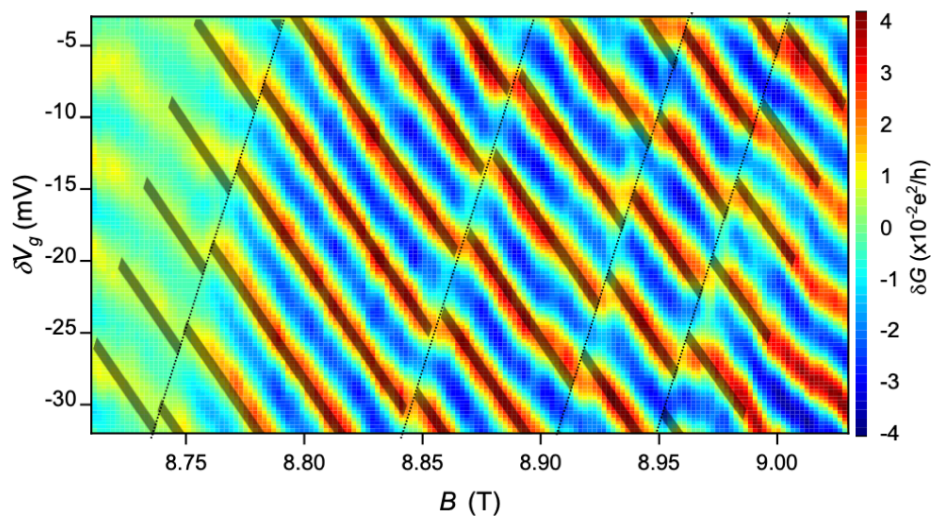

Figure 1: (a) Gate-defined quantum dot and measurement set-up with schematic of interfering edge states weakly tunneling across two constrictions, surrounding localized quasiparticles (spirals). The edge-state sketch, for filling factor $\nu \sim 1 / 3$ in the dot and bulk, and $\nu \lesssim 1 / 3$ in the constrictions, is superimposed on an electron micrograph showing gate pattern and device size. An additional gate over the center of the dot, which remained at zero volts, is not shown. (b) Color map of device conductance variation $\delta G$ (with a smooth background subtracted from total conductance) as a function of magnetic field, $B$, and voltage on a side-gate, $\delta V_{g}$. Conductance features moving downward with increasing field indicate Aharonov-Bohm interference. Jumps between the stripes, associated with charge instability in the device, are roughly one third of the spacing between stripes. The jump by one-third of the spacing is the signature of factional statistics. Both (a) and (b) are from Ref. [7].

nonabelian braiding, and more complex devices in which interior quasiparticles are not only added and subtracted on demand, but also moved around [9].

\section{References}

[1] R. B. Laughlin, Phys. Rev. Lett. 50, 1395-1398 (1983)

[2] D. Arovas, J. R. Schrieffer, and F. Wilczek, Phys. Rev. Lett. 53, 722 (1984)

[3] This experiment is discussed in the Recommendation of S. Girvin, "A collider for anyons", Journal Club for Condensed Matter Physics, July 2020. DOI: 10.36471/JCCM_July_2020_01.

[4] C. de C, Chamon, D.E. Freed, S. A. Kivelson, S. L. Sondhi, and X. G. Wen, Phys. Rev. B 55, 2331 (1997)

[5] Experiment: Y. Zhang, et al. Phys. Rev. B 79, 241304 (2009), N. Ofek, et al., PNAS, 107, 5276 (2010); Theory: B. I. Halperin, A. Stern, I. Neder, and B. Rosenow, Phys. Rev. B 83, 155440 (2011).

[6] J. Nakamura, et al., Nature Physics, 15, 563-569 (2019). 
[7] J. Nakamura, S. Liang, G. C. Gardner, and M. J. Manfra, arXiv::2006.14115 (2020).

[8] Screening gate method: A. Kou et al. Phys. Rev. Lett. 108, 256803 (2012); Air bridge method: N. Ofek, et al., PNAS, 107, 5276 (2010).

[9] S. Das Sarma, M. Freedman, and C. Nayak, Phys. Rev. Lett. 94, 166802 (2005). 